\title{
Organogénesis del sistema digestivo del pez Pterophylum scalare (Perciformes: Cichlidae)
}

\author{
Mireya Ávila Botello ${ }^{1}$, Ivette insuasty León ${ }^{2} \&$ Edilma Guevara Rozo ${ }^{3}$ \\ 1. Biología Universidad Distrital Francisco José de Caldas 2007, Bogotá Colombia, mireyavilab@yahoo.com \\ 2. Biología, Universidad Distrital Francisco José de Caldas 2007, Bogotá Colombia, ivetteinsuasty@yahoo.com \\ 3. Biología, Pontificia Universidad Javeriana, Bogotá, Colombia; guevarae@javeriana.edu.co
}

Recibido 05-II-2008. Corregido 30-VI-2008. Aceptado 31-VII-2008.

\begin{abstract}
Organogenesis of the digestive system of the fish Pterophylum scalare (Perciformes: Cichlidae). There is little knowledge on the development of the angelfish Pterophyllum scalare (Liechtenstein 1823), a species of economical and biological value for inland water ecosystems. We recorded net development time of each organogenetic stage, cumulative time and characteristic structure differentiation for each stage. We found eight organogenetic stages for the digestive system, between the gastrula and the total re-adsorption of the vitelin sack. The total time for the organogenetic development of the digestive system was 119 hours and 44 minutes. Rev. Biol. Trop. 56 (4): 1857-1870. Epub 2008 December 12.
\end{abstract}

Keywords: organogenesis, stages, digestive system, embrionary development, cellular differentiation, fish.

El escalar es una especie tropical de la región de la orinoquía colombiana. Puede llegar a los $15 \mathrm{~cm}$ de longitud y se caracteriza por su esbeltez lateral; las aletas dorsal y anal son muy grandes y forman un triángulo en el aspecto general del pez. Los escalares no son peces que presenten diferencias apreciables entre los machos y las hembras. La aleta caudal también es grande, y las abdominales se han convertido en dos radios largos de hasta $8 \mathrm{~cm}$. Su temperatura está entre 22 y $30^{\circ} \mathrm{C}$. El escalar se alimenta de presas vivas, incluyendo alevines. Los individuos se escalonan de acuerdo con el nivel de dominancia que tienen. El ejemplar más grande y saludable suele ser el pez dominante que compite por los espacios, por la comida y por la pareja reproductora. Se enfrentan con las aletas muy abiertas (Petrovicky 1990). Las peleas aumentan en intensidad en la época de reproducción. Se dan cuando se forman las parejas, cuando la pareja delimita su territorio, y sobre todo cuando la pareja defiende la puesta. Sólo las hembras que estén también dispuestas para la reproducción aceptan los ataques del macho, que nunca llegan a consumarse. Solo la hembra elegida penetra el territorio. En el momento del desove, el macho presenta un espermiducto corto, terminado en punta ligeramente inclinado hacia delante y en la hembra se observa un oviducto un poco más largo, grueso y redondeado, inclinado hacia atrás (Verhoef 2002). Limpian una superficie plana para depositar los huevos; normalmente una hoja grande, una piedra plana o incluso un lado del acuario. Suelen depositar más de 200 huevos; si son pequeñas, alrededor de 150. Ejemplares muy desarrollados pueden desovar hasta 350 huevos (Axelrod 1978).

Este trabajo se enfocaen la organogénesis: tiempo neto de desarrollo de cada estadio de la organogénesis, tiempo acumulado, y diferenciación de estructuras características de cada estadio. 


\section{MATERIALES Y MÉTODOS}

Se utilizaron siete posturas de escalares (Pterophyllum scalare, Liechtenstein 1823), obtenidas en la Universidad de los Llanos (IALL), y dos posturas obtenidas en los estanques de Oranda Fish (Acacias, Meta). Las parejas seleccionadas fueron ubicadas dentro de acuarios y la reproducción se llevó a cabo de forma natural Se retiraron ocho ovocitos por cada muestreo con una micropipeta, fueron observadas al estereoscopio. Las ovoposiciones se colocaron dentro de la solución fijadora a una temperatura de $14^{\circ} \mathrm{c}$ por un intervalo de tiempo máximo de 15 días; se dio comienzo al proceso de deshidratación e inclusión planteado por Cubillos (1994). Luego se realizaron los montajes y cortes histológicos de 5 micras de grosor y la correspondiente tinción hematoxilina -eosina, el cual se inició con la desparafinización del tejido y luego se sumergió en una serie ascendente de alcoholes para finalmente montar la laminilla y realzar la lectura histológica al microscopio

\section{RESULTADOS}

Se identificaron ocho estadios. Para establecer cada estadio se tomo como base la tabla de desarrollo en Pterophyllum scalare, descrita por Cubillos (1994).

Estadio \# 1. desde gástrula hasta la pre-eclosión tiempo: de $\mathbf{0 . 0 0}$ a 34 horas: 37 minutos: $\mathrm{Al}$ inicio son las regiones presuntivas ectodérmicas las que migran hacia el interior del blastodisco para determinar cual será la parte posterior del embrión. A medida que las células ectodermales siguen su desplazamiento hacia el centro en la línea media anterior, se forma el cordomesodermo. Las diferentes clases de mesodermo de la parte anterior del embrión se invaginan de la misma manera, dando como resultado la curvatura del blastodisco. Aunque no se forma arquenterón debe considerarse que el inicio de la invaginación de las células corresponde al borde del blastoporo, el cual es el camino por donde el endodermo y el mesodermo pasan hacia el interior del embrión. Se identificaron las tres capas germinales: ectodermo, mesodermo y endodermo. En donde esta ultima dará origen al sistema digestivo. Cuando el embrión alcanza entre 24 a 26 pares de somitas, en corte transversal a nivel de médula, en la región dorso lateral del embrión, se advierte una masa de células mesenquimáticas que darán origen en subsecuentes estadios al sistema digestivo. Cerca de estas células en diferenciación se observa la presencia de los túbulos pronefricos. (fig. 1).

Estadio \# 2. Faringe: comprende desde eclosión hasta reabsorción del saco vitelino tiempo: de 34 horas 37 minutos a 154 horas minutos: Desde el estadio de gástrula hasta el de pre - eclosión, el embrión presento una serie de contracciones, generando el desprendimiento del corion y por tanto la correspondiente eclosión; momento en el cual, se observa en corte transversal a la altura del mielencéfalo, los arcos faríngeos acompañados de una fisura en la región ventral que corresponde al inicio de la faringe. Dicha fisura se caracteriza por estar tapizada en su mayoría por células epiteliales cúbicas. (fig. 2).

Externamente en el embrión se puede observar región cefálica y región caudal. Además, se observa que la circulación corporal se concentra principalmente en la región media ventral del embrión en limites con el vítelo. En la región cefálica se evidencia la formación de 6 órganos fijadores organizados en parejas los cuales utiliza para adherirse al sustrato (fig. 3).

A la altura del mielencéfalo durante la formación del esbozo externo de branquias y de la aleta pectoral, se presenta debajo de la notocorda la faringe con mayor diámetro y en la mitad del esbozo de los arcos branquiales. Las células planas características de la faringe al inicio de este estadio cambian progresivamente a células cúbicas con núcleos centrales), y a medida que se diferencian los filamentos branquiales las células que tapizan la faringe se tornan cilíndricas con núcleos básales (fig. 4).

Cuando habita la reabsorción total del saco vitelino los cortes transversales a la altura de 


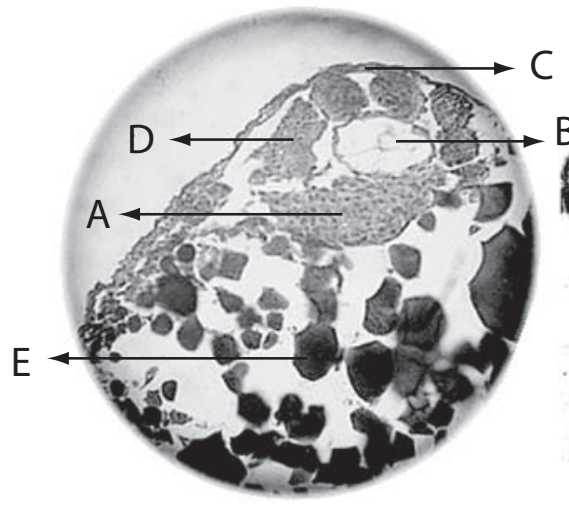

Figura 1

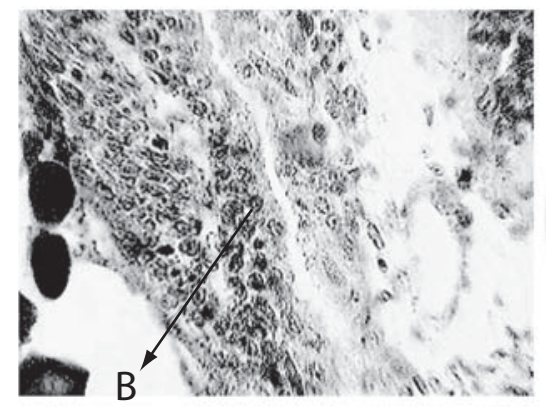

Figura $2 b$

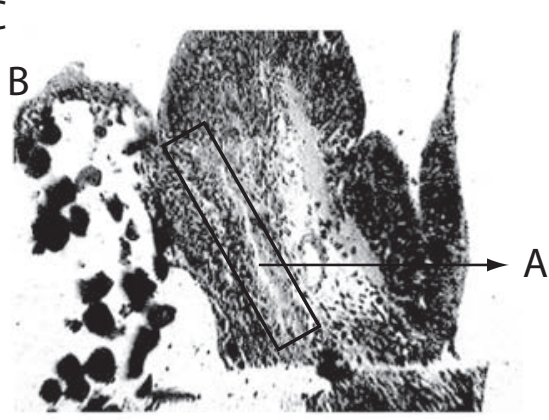

Figura $2 a$

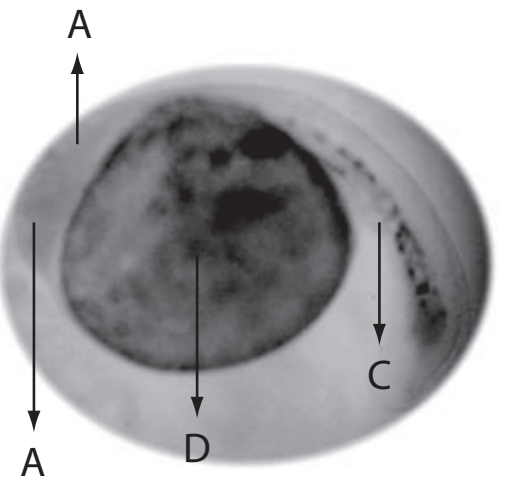

Figura 3

Fig. 1. Corte histológico a nivel de medula espinal. A. Células mesenquimáticas. B. Notocorda. C. Medula espinal. D. Somitas. E. Vítelo. Aumento $40 \mathrm{x}$.

Fig. 2. Corte transversal a nivel de mielencéfalo. A. esbozo de faringe. B. Células epiteliales de revestimiento. Aumento 40 $\mathrm{x}$ y $100 \mathrm{x}$.

Fig. 3. Embrión en toto. A. Órganos fijadores B. Otocistos C. Circulación Caudal D. Vitelo.

mielencéfalo muestran pliegues de la faringe formadas por un epitelio cilíndrico simple, como tejido de revestimiento definitivo para esta cavidad.

Este estadio dura en promedio: 119 horas y 44 minutos.

Estadio \# 3. Esbozo de maxila y premaxila. desde botón de aletas pectorales hasta reabsorción total del saco vitelino. Tiempo: 47 horas 47 minutos a 154 horas 21 minutos. En este estadio se evidencia mayor desarrollo del embrión, a nivel del mielencéfalo se encuentran tres arcos branquiales diferenciados: el primero localizado anterior al otocisto, el segundo debajo del otocisto y el tercero detrás del mismo. Del primer arco branquial se forma el esbozo de mandíbula. Se diferencia como una masa de células situadas detrás del ojo, es decir entre el ojo y la primera bolsa branquial, esta masa de células se convierte en el primer arco mandibular, cuya parte inferior dará origen a la mandíbula. El primordio de su formación se presenta como una condensación celular de forma piramidal. En cortes histológicos se puede apreciar en la región posterior, un 
mayor desarrollo de la mandíbula y al mismo tiempo el desarrollo interno del cartílago perteneciente a maxila y premaxila (fig. 5).

En el momento de la reabsorción total del saco vitelino, la boca esta conformada por la mandíbula bien desarrollada y la maxila y premaxila en diferenciación. La maxila y premaxila se diferencian a partir de la porción dorsal del primer arco branquial. La mandíbula posee una forma de tabique y se extiende ventralmente desde la parte anterior de la cúpula óptica hasta la parte ventral del otocisto. La mandíbula esta irrigada por vasos sanguíneos.

Este estadio dura en promedio: 106 horas y 34 minutos.
Estadio \# 4 el esófago. desde botón de aleta pectoral hasta la reabsorción total del saco vitelino tiempo: 53 horas 10 minutos a 154 horas 21 minutos. A nivel de mielencéfalo, se observa que la luz de la faringe mantiene una forma lineal y progresivamente dicha luz adquiere forma redondeada lo cual determina el inicio de la formación del esófago; las células del esófago empiezan un progresivo desarrollo adquiriendo forma alargada y núcleos dirigidos hacia la membrana basal, conformando un epitelio estratificado plano no queratinizado. Durante el esbozo de maxila y premaxila, el esófago presenta una serie de pliegues formados por un epitelio estratificado

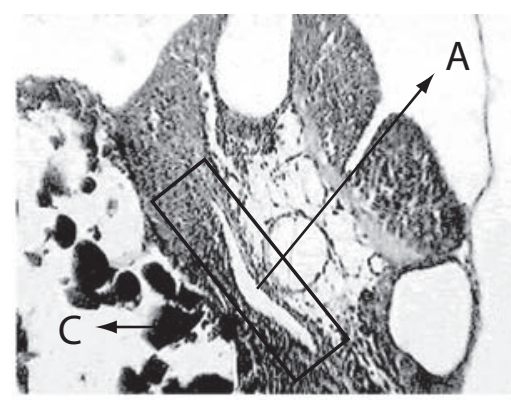

Figura $4 a$

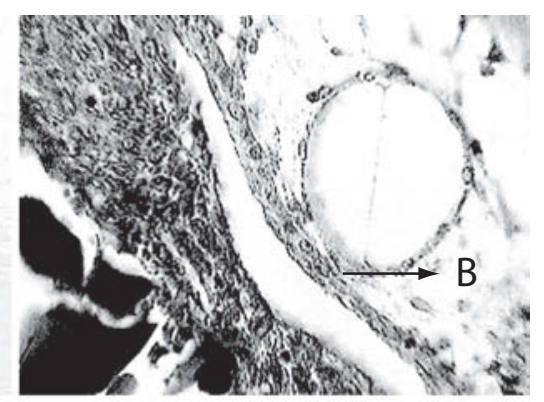

Figura $4 b$

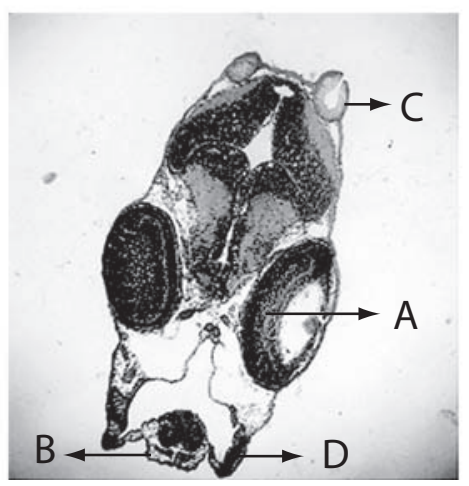

Figura 5

Fig. 4a y 4b Corte transversal a nivel de mielencéfalo. A faringe B Células cúbicas C.Vitelo. Aumento 40 x y 100 x.

Fig. 5 Corte transversal a nivel de diencéfalo. A. cúpula óptica B. Esbozo de maxila y premaxila C. Balancines D. Cartílago de maxila y premaxila. Aumento 10x. 
plano no queratinizado, rodeado por una capa de tejido conectivo laxo que forma parte de la capa submucosa y por encima de esta un grupo de células musculares que corresponden a los mioblastos que participan en la formación de la túnica muscular. Entre el estadio de movimiento mandibular y el momento de la reabsorción total del saco vitelino los mioblastos hasta ahora presentes en la túnica muscular, se observan - en la zona anterior del esófago y se transforman en células musculares estriadas y en la zona posterior del esófago los mioblastos se transforman en células musculares lisas. (fig. 6).

Este estadio dura en promedio: 101 horas y 11 minutos.
Estadio \# 5. Diferenciación del hígado. desde el botón de la aleta pectoral hasta reabsorción total del saco vitelino. Tiempo: 53 horas 10 minutos a 154 horas 21 minutos. El hígado de Pterophyllum scalare, comienza a diferenciarse a la altura del intestino posterior, por debajo de la vejiga natatoria y de las asas intestinales. Puede identificarse en límites con el vítelo. La presencia de cordones hepáticos epiteliales cuyos núcleos son redondos, presentan un aspecto uniforme, y se forma una red trabecular que hace parte del parénquima del hígado. Durante la formación de filamentos branquiales, el hígado se observa bastante voluminoso y los hepatocitos están totalmente diferenciados y ubicados en forma radial. Es

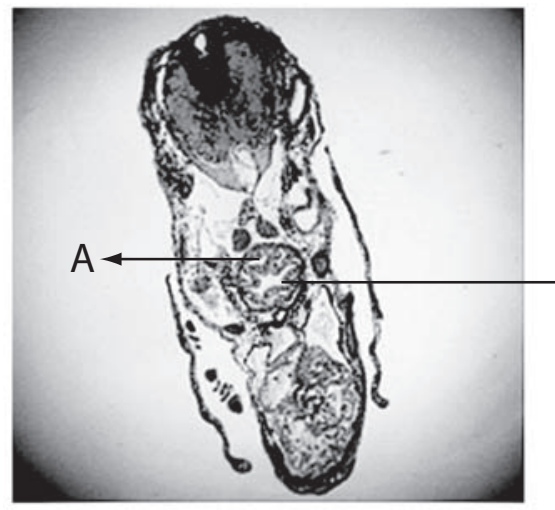

Figura 6a

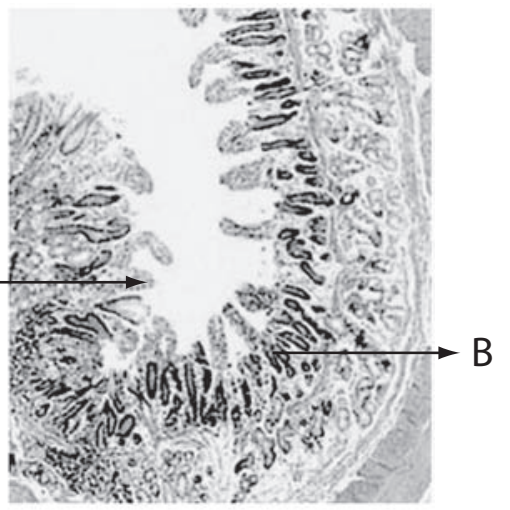

Figura $6 \mathrm{~b}$

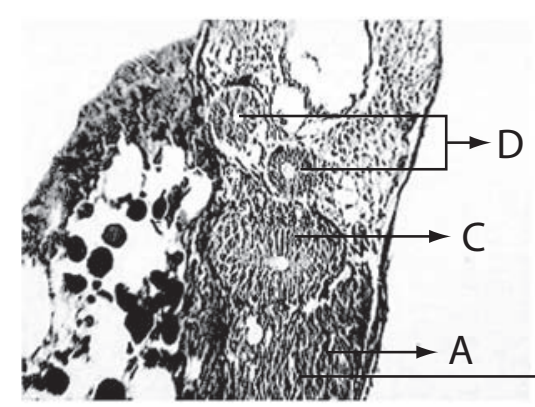

Figura 7a

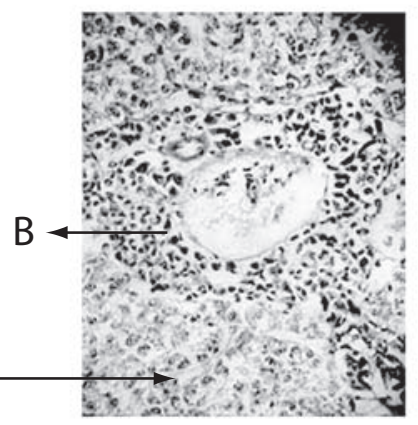

Figura $7 b$

Fig. 6a y 6b. Corte transversal a nivel de medula espinal. A. Esófago B. Células epiteliales de revestimiento. Aumento 10 $\mathrm{x}$ y $100 \mathrm{x}$

Fig. 7a y 7b Corte transversal a nivel de medula espinal. A. hígado. B. hepatocitos C. Asa intestinal. D. Pronefros. 
importante resaltar que entre hígado y páncreas no fue posible establecer diferencias celulares. (fig. 7). Cuando se absorbe el saco vitelino, la porción hepática esta presente con su composición trabecular característica.

Este estadio dura en promedio: 101 horas y 11 minutos.

Estadio \# 6 intestino. desde eclosión hasta reabsorción total del saco vitelino. Tiempo 34 horas 37 minutos a 154 horas 21minutos: Desde el estadio de faringe, se pudo observar la primera asa intestinal, en la cual se puede ver secuencialmente que los núcleos celulares que no se han ubicado hacia la base, se encuentran alternos dando la apariencia de un epitelio seudo estratificado (fig. 8).

A medida que la altura del corte es mayor, estos se van ubicando hacia la base de las células buscando conformar lo que será el intestino posterior. En corte transversal a nivel de la medula se ve claramente la presencia de los túbulos pronefricos a cada lado del asa intestinal. (fig. 9).

Durante el momento de la eclosión en el estadio de la faringe y simultáneamente

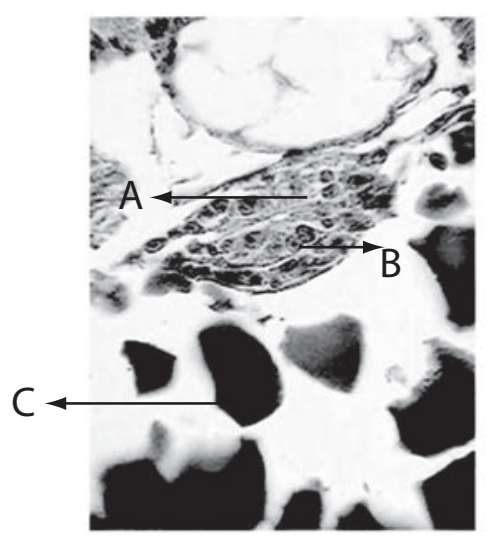

Figura 8

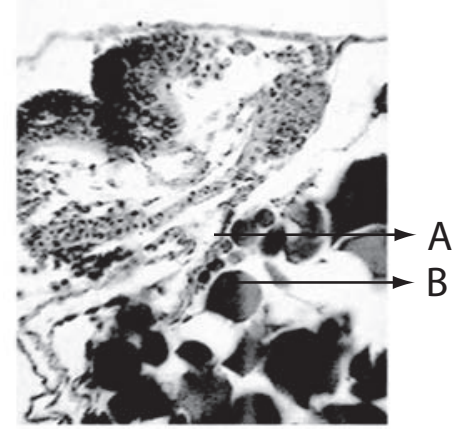

Figura 10

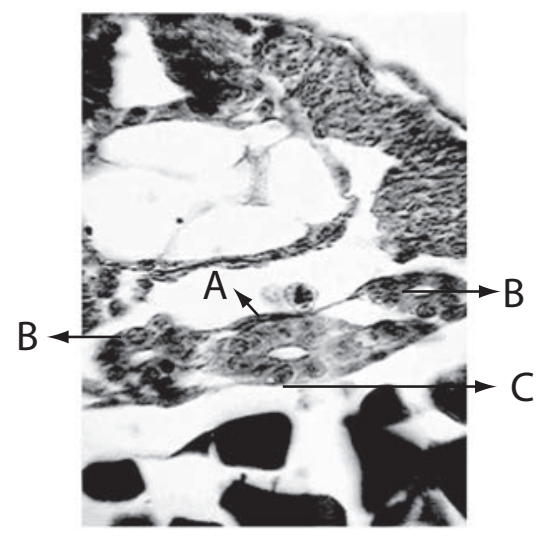

Figura 9

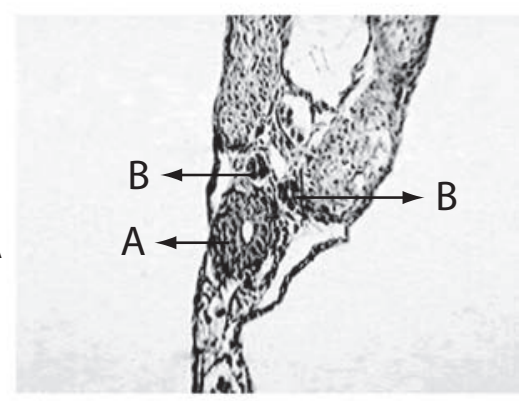

Figura 11

Fig. 8 Corte transversal a nivel de medula espinal. A. Asa intestinal. B. Tejido epitelial de revestimiento C. Vitelo

Fig. 9 Corte transversal a nivel de medula espinal. A. Asa intestinal. B. pronefros. C. núcleos ubicados hacia la base (intestino posterior)

Fig. 10 Corte transversal a nivel de mielencéfalo. A. Intestino medio en relación con el vitelo B Vitelo

Fig. 11 Corte transversal a nivel de medula espinal. A. asa intestinal con núcleos básales B. Pronefros. 
con la formación del intestino anterior se puede apreciar en cortes histológicos un grupo de células sobre la región media-ventral del embrión, que empiezan a caracterizarse por la presencia de núcleos básales más grandes y más coloreados. Diferencias que marcan la zonificación del intestino anterior y medio, en donde este último da paso a la diferenciación del intestino posterior.

Además identifica la posición del intestino medio está relacionada con la formación del saco vitelino (fig. 10).

Durante el esbozo de branquias luego de marcarse la zonificación del intestino anterior y medio, este último dio paso a la diferenciación del intestino posterior, el cual se pudo apreciar en los cortes histológicos por la formación clara de una gran asa intestinal a la altura de la medula, con núcleos básales grandes, esta asa intestinal se ubica en la mitad de los pronefros (fig. 11), y se hace muy notable las diferencias existentes entre el intestino anterior y el intestino medio debido a que las células aumentan secuencialmente de tamaño de acuerdo a la región donde se encuentren ubicadas.

Durante la formación de la vejiga natatoria que es una estructura en forma de saco, se puede observar externamente que el ducto digestivo se abre hacia el exterior, puesto que el embrión presenta una dilatación al final del intestino posterior marcada en la región del proctodeo en la cual se sitúa la membrana anal que termina por romperse, dejando paso libre entre el tubo digestivo y el exterior (fig. 12).

En cortes histológicos en la porción terminal del intestino posterior se aprecia un grupo de células epiteliales cilíndricas formando un anillo estrecho que corresponde a la región anal (fig. 13).

Finalizando este estadio la zona del intestino medio tiende a desaparecer puesto que pierde conexión con el saco vitelino ya que este es absorbido y el intestino queda revestido por un tejido epitelial simple cilíndrico. A nivel de medula espinal se observa un grupo de asas intestinales cada una formada por una capa mucosa y una capa muscular (fig. 14).

Este estadio dura en promedio: 119 horas y 44 minutos.
Estadio \# 7. Estomago. desde esbozo externo de maxila y premaxila hasta reabsorción total del saco vitelino. Tiempo: 66 horas 01 minutos a 154 horas 21 minutos. En corte transversal a nivel de medula, se puede observar al seguir la secuencia de los cortes histológicos, que la luz del esófago mantiene su forma redondeada y la conformación de las capas que lo caracterizan permanece igual, sin embargo la túnica muscular que en el esófago esta conformada por células que harán parte del músculo esquelético en la parte anterior, cambia progresivamente y se manifiestan fibras musculares lisas en la parte posterior en disposición circular, lo cual indica que se ha iniciado la formación del estomago. Durante el estadio de movimiento mandibular, las células del estomago comienzan a alargarse y sus núcleos se dirigen más hacia la membrana basal. Las células anteriormente mencionadas poco a poco conforman la túnica mucosa en forma de pliegues, con un epitelio de revestimiento simple cilíndrico. Finalmente durante la reabsorción del saco vitelino el estomago queda revestido por un epitelio simple cilíndrico. Presenta una capa muscular, posterior a la lámina propia constituida por mioblastos en disposición circular. (fig. 15)

Este estadio dura en promedio: 88 horas y 20 minutos.

Estadio \# 8 movimiento mandibular. desde aparición de filamentos branquiales y movimiento mandibular hasta reabsorción total del saco vitelino. Tiempo: 79 horas 16 minutos a 154 horas 21 minutos: Hacia la región mandibular el embrión intenta una serie de movimientos que darán paso al movimiento mandibular y por consecuencia a la apertura de la boca (fig. 16).

Histológicamente el aparato bucal conformado por mandíbula, maxila y premaxila muestra un mayor grado de osificación, se rompe la membrana faríngea y la mandíbula adquiere movimiento (fig. 17). Mientras tanto los filamentos branquiales se diferencian como condensaciones celulares de forma piramidal localizadas sobre cada uno de los arcos 


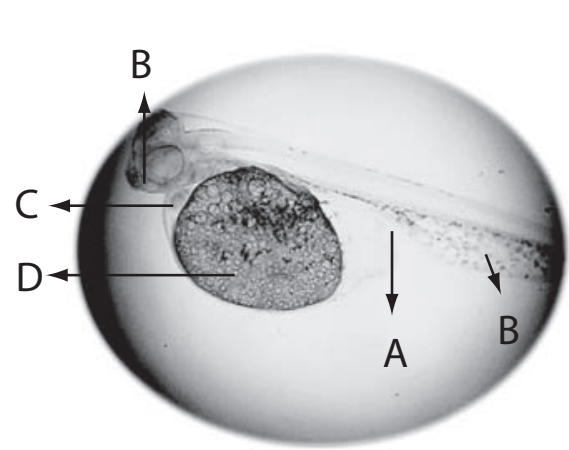

Figura 12

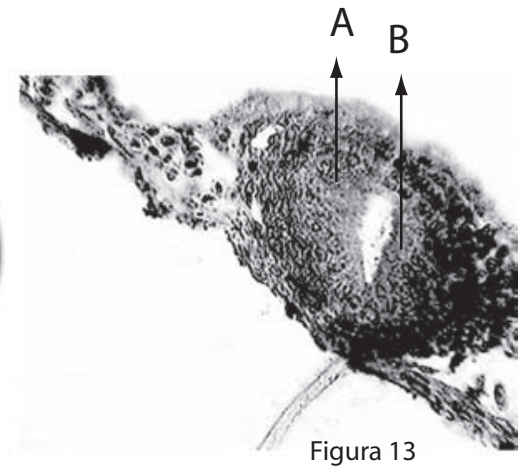

Figura 13

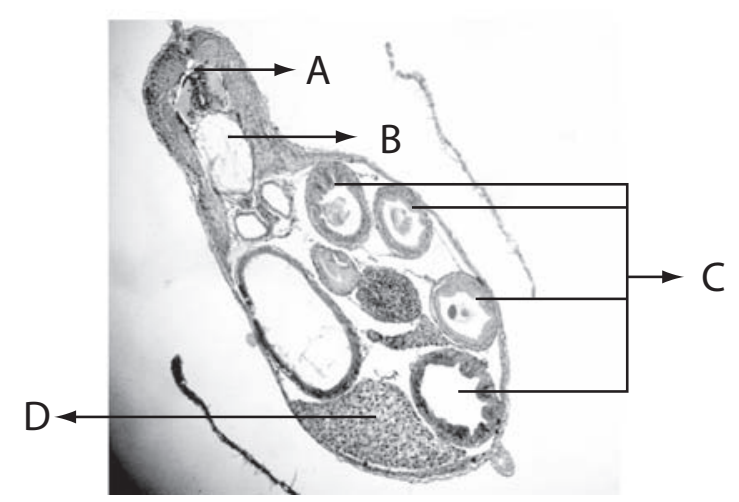

Figura 14

Fig. 12 Embrión en toto. A. Obsérvese la apertura externa del intestino hacia la región del proctodeo. B. Región cefálica. C. Corazón tubular. D Vitelo. E. Región caudal. Aumento 40x

Fig. 13 Corte transversal a nivel de medula espinal. A. asa intestinal. B. células epiteliales cilíndricas

Fig. 14 Corte transversal a nivel de medula espinal. A. Médula B. Notocorda. C. Asas intestinales. D. Hígado.

branquiales y cubiertos por un opérculo cartilaginoso. Este estadio dura en promedio: 75 horas y 5 minutos.

\section{DISCUSIÓN}

Se reportaron un total de 8 estadios para la organogénesis del sistema digestivo, que van desde el inicio del estadio de gástrula hasta el estadio de movimiento mandibular, teniendo en cuenta algunos aspectos morfológicos de la especie y las características propias del sistema digestivo. Todos los estadios se describieron hasta la reabsorción total del saco vitelino, momento en el cualculmina el desarrollo embrionario (Gueimundi 1989 y Cubillos 1994). Los individuos en este estadio han desarrollado las características morfológicas y fisiológicas para llevar una vida independiente. Es importante destacar en este trabajo que los órganos que conforman el sistema digestivo se van constituyendo paulatinamente y su origen es de la capa germinativa endodérmica con la contribución parcial del ectodermo y el mesodermo (Gueimundi 1989). Una vez ocurrida la fertilización las primeras etapas corresponden a clivaje y blástula y luego se realiza la etapa de gástrula en la cual se inicia la formación del 


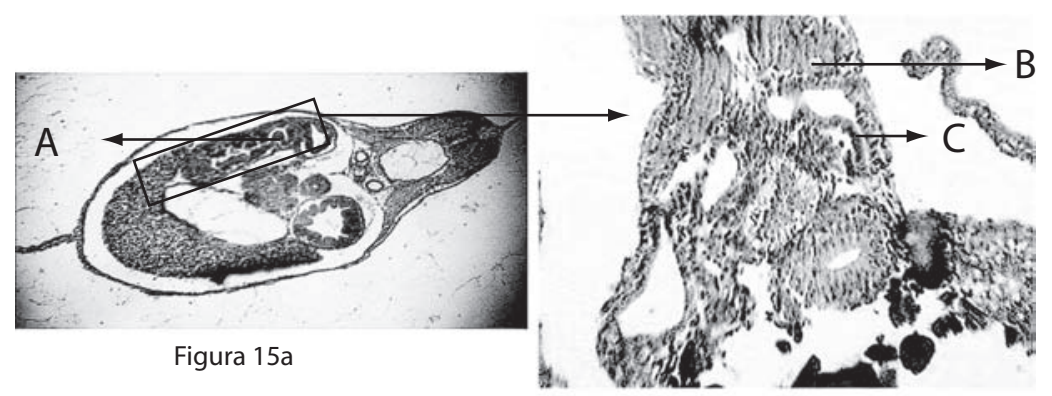

Figura 15b

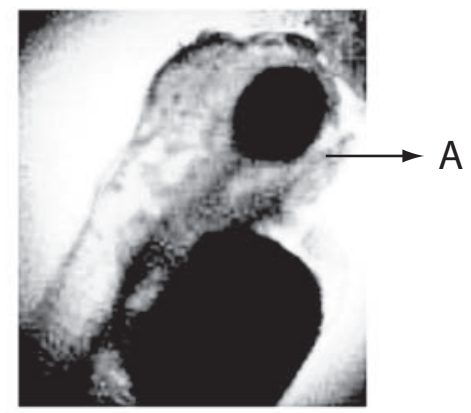

Figura 16

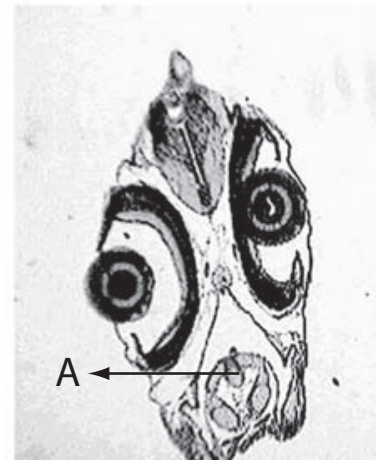

Figura 17

Fig. 15 a y 15 b Corte transversal a nivel de medula espinal. A. estómago B. Epitelio cilíndrico simple C. Túnica mucosa

Fig. 16. Embrión en toto. A. Movimiento mandibular (Ruptura de la membrana bucal).

Fig. 17. Corte transversal a nivel de diencéfalo A. Osificación de mandíbula y maxila

sistema digestivo, parte fundamental de este trabajo.

Gastrulación: En esta etapa se originan las capas germinativas (Theodore 1978). Cuando el individuo en formación entra a la fase de gastrulación, el extremo posterior del blastodisco aumenta su espesor y todo el borde se engrosa y constituye el anillo germinal. Una de las características que definen la gastrulación, son los movimientos morfogéneticos, (invaginación, epibolia, delaminación, etc) la epibolia o recubrimiento en Pterophyllum scalare, se observa claramente por el desplazamiento de una estructura denominada anillo germinal, que circunda toda la masa en movimiento. El movimiento de invaginación se evidencia por la migración hacia el interior del embrión de un grupo determinado de células, las cuales más adelante darán origen a la notocorda. Dado que la mayoría de las manifestaciones morfológicas para la formación de la gástrula ocurren en todo el borde del disco embrionario, este mismo borde podrá considerarse como análogo a los labios dorsal, lateral y ventral del blastoporo del anfibio (Pizano 1981). Es importante resaltar que el destino del blastoporo esta relacionado con la formación del ano, el borde que rodea al vítelo corresponde a los labios del blastoporo.

En un periodo posterior el blastoporo se cierra por constricción de sus labios en la región posterocaudal; antes que se cierre completamente, se puede observar un tapón de 
vítelo proyectándose entre sus bordes o labios. Gueimundi 1989, indica que en la mayoría de las especies se encuentra bien cubierto, pero en Pterophyllum scalare esta característica no corresponde a este estadio porque, cuando se cierra el blastoporo los embriones se encuentran en un periodo más avanzado de desarrollo. Balinsky 1978, reporto que durante la gastrulación se forman las tres hojas germinales lo cual permite que se inicie el proceso de diferenciación del embrión, con destinos específicos, dando lugar a la formación de tejidos y órganos es decir la organogénesis. Las masas continuas de células de las tres hojas germinales forman tres grupos menores de células, cada uno de los cuales esta destinado a producir un órgano o una parte especifica del individuo en formación. De acuerdo como se describe en el estadio 1 se puede apreciar en cortes transversales una agrupación de células mesenquimáticas bajo la notocorda en la región dorso lateral las cuales (Theodore 1978), se reúnen para formar masas densas, sin constituir un epitelio como tal, estás células mas adelante se diferenciaran en tejidos: muscular, óseo y cartilaginoso. Se pudo confirmar que las células mesenquimáticas se concentran cerca de la superficie del epitelio o alrededor de algún órgano epitelial, tal como sucede con el tubo digestivo, para formar las capas restantes de naturaleza conjuntiva y muscular. Además (Cetinkaya 1999) en Chalcalbumus Tarichi reporto que las células mesenquimáticas tienen la propiedad de migrar, con destinos diferentes para dar lugar a la formación de los esbozos de los órganos. Balinsky en 1978 indico que las células mesenquimáticas pueden originarse de la descomposición de capas epiteliales el cual es considerado uno de los procesos más importantes puesto que suministra los materiales para el ulterior desarrollo de los esbozos de órganos como cavidad bucal, esófago e intestino.

Eclosión: En Pterophyllum scalare, al momento de la eclosión el vítelo se encuentra rodeando el tubo intestinal y la región media ventral del intestino primitivo presenta sus paredes incompletas y comunicadas con el saco vitelino lo cual coincide con lo reportado para Chalcalburnus tarichi (ÇETNKAYA 1999).

Intestino: En el intestino primitivo del embrión se distinguen tres regiones básicas: El intestino anterior, medio y posterior. Gueimundi 1989, describió que el intestino anterior de los teleósteos va desde la región del estomodeo hasta la zona hepática. Esta región origina la boca, el área faríngea, el esófago, el estomago. Además se origina la región pancreática. El intestino medio, situado entre el intestino anterior y el intestino posterior, origina la mayor parte del intestino delgado. En periodos tempranos esta zona esta relacionada con la formación del saco vitelino. El intestino posterior origina el resto de las estructuras del tubo intestinal; el intestino primitivo se extiende durante un tiempo hacia la región caudal y constituye el intestino caudal o potsanal que desaparece después. Para la formación del estomodeo la región anterior, el ectodermo, forma una invaginación, que se dirige hacia una evaginación oral del intestino anterior y constituye una doble membrana que se denomina membrana oral o faríngea. Cuando esta membrana se rompe, se fusiona con el ectodermo, dando como resultado una estructura continua. En la región caudal del embrión el endodermo forma el proctodeo, el cual se une a una evaginación ventral del intestino posterior y forma la membrana cloacal que termina por romperse y dejar el paso libre entre el tubo digestivo y el exterior. Paniagua en 1983 reporto que en los vertebrados se pueden distinguir un intestino anterior, medio y posterior, el anterior esta integrado por la boca, faringe y estomago, el intestino medio suele denominarse intestino delgado y el posterior intestino grueso. Además, Anzola en 1993 indico que el sistema digestivo de los peces se compone básicamente, al igual que todos los vertebrados, de boca, faringe, esófago, estomago, intestino, ano. Como órganos anexos están: El hígado, vesícula biliar y páncreas.

En cuanto al intestino (Halver 1989 y Smith 1989), reportan en peces teleósteos dos regiones intestinales, una región anterior y una posterior. En Pterophyllum scalare se encontró 
una diferenciación microscópica en el intestino que permite establecer regiones diferentes del intestino. Al igual que Arias 1996, en Sapura se reconocen morfológicamente diferentes regiones. Smith 1989, reporta en peces teleósteos diferencias entre la región media y la región anterior. En Pterophyllum scalare fue posible observar las tres regiones del intestino: Intestino anterior, intestino medio e intestino posterior. Al igual que lo reportado en la Cachama Blanca Sp: Piaractus brachipomus, se puede establecer en Pterophyllum scalare diferencias en cuanto a la presencia de pliegues en el intestino medio que se hacen cada vez menos frecuentes y de menor tamaño hasta desaparecer en el intestino posterior.

En el intestino de Pterophyllum scalare el número de vellosidades de la mucosa se presentan, principalmente en la región pilorica se observan largas y delgadas y a medida que se acercan al ano aumentan su grosor y disminuyen en número. No se observa un cambio en su epitelio que es cilíndrico simple en todo el tracto intestinal. En Pterophyllum scalare, las vellosidades presentan lámina propia y un epitelio cilíndrico simple similar a lo reportado para mamíferos (Halver en 1989), sin embargo Velandia 2000 y Caceci 1984, no aceptan que los peces presenten vellosidades con la misma morfología que presentan los mamíferos

Faringe: En Pterophyllum scalare, la faringe conecta la cavidad bucal con el tubo digestivo, principalmente con el esófago. Histológicamente las vellosidades de la faringe, desempeñan un papel importante ya que al pasar partículas grandes de alimento, estas se dilatan previniendo el desgaste de la mucosa, la presencia de músculo estriado permite tener control sobre el alimento. En Pterophyllum scalare (Liechtenstein, 1823) la faringe en cortes transversales se observo en el Estadio \# 2 revestida de un epitelio formado de células de forma cúbica.

Esófago: Florez 2000, reporta que histológicamente el esófago en la Sp: Trichopterus trichopterus, presenta una mucosa con un epitelio cilíndrico simple, sin embargo en $\mathrm{Sp}$ : Pterophyllum scalare el esófago esta formado por un epitelio estratificado plano no queratinizado soportado por una matriz elaborada por fibroblastos que posteriormente constituyen la submucosa de tejido conectivo laxo areolar. También se observaron mioblastos que posteriormente forman la túnica muscular constituida por músculo esquelético, características que también están presentes en la Sp: Trichopterus trichopterus, y que fueron reportadas por Florez 2000. Clark y Witcomb 1980 y Halver 1989 , plantean que la gran mayoría de los peces presentan esófago corto y ancho, con secreción de mucus en la vía de entrada al estomago; la mucosa incluye capas de músculo liso amplias y cerca del estomago. IRRI 1999 reporto la misma observación en la perca y estableció que no hay actividad enzimática, excepto el ácido fosfático en la superficie del epitelio. Paniagua 1983 indica que en los teleósteos las células de revestimiento epitelial interno del esófago no son primordialmente secretoras, mas bien forman un epitelio resistente al desgaste. En peces adultos como los cyprinidos se presenta una capa muscular llamada musculares mucosae formada de músculo esquelético; en este trabajo se hace referencia a mioblastos y no a un tejido muscular totalmente caracterizado. En 1984 Chávez y Vazzoler reporta para Semaprochilodus insignis que presenta un esófago constituido por dos regiones muy diferentes. La región anterior revestida por epitelio estratificado pavimentoso y la región posterior constituida por un epitelio cilíndrico simple, cuyas células son de características secretoras. Florez 2000 reporto para T. trichopterus, un epitelio cilíndrico simple en todo su recorrido lo cual significa que el epitelio y las glándulas mucosas tienen como única función protección, lubricación y conducción del alimento por el esófago. . Esta característica no coincide con lo reportado para la Sp: Pterophyllum scalare. En cuanto a esófago y estómago en este trabajo hacemos referencia a mioblastos y no a capa muscular como tal, debido a que al estar el pez en estado embrionario el tejido muscular se encuentra aún en estado de desarrollo. 
Hígado y páncreas: Ziswiler 1980, plantea que el hígado en los peces suele ser voluminoso y localizado centralmente, lo cual coincide con lo observado para Pterophyllum scalare, además Ziswiler agrega que el hígado actúa sobre todo como acumulador de glucógeno, se encarga de la producción de bilis y de grasa. Flores 2000 reporta para el hígado de T. trichopterus, hepatocitos ubicados en forma radial en cordones de dos células de espesor, separados por sinusoides que confluyen hacia una vena central, Paniagua 1983 y Ulrich y Storch 1973 reportan la misma morfología para peces teleósteos, sin embargo en Pterophyllum scalare, el parénquima del hígado se conforma por células uniformes en todo el órgano por lo que no pudieron ser fácilmente identificables las subunidades estructurales. En la carpa Cyprinius carpio, el hígado esta integrado con el páncreas y no presenta lóbulos. En este trabajo se deja la posibilidad de que en Pterophyllum scalare este formado un hepatopancreas como también lo afirma Flores 2000 para T. trichopterus, puesto que no fue posible observar diferencias celulares características del páncreas, lo cual puede ser también resultado del corto tiempo de desarrollo de los embriones observados.

Estomago: En cuanto a la formación de estomago, se presento como una dilatación del tubo digestivo que almacena y digiere parte del alimento. Werner 1987 plantea que desde el punto de vista de la fisiología de la nutrición pueden clasificarse en dos grupos: peces con estomago y peces sin estomago. Zihler 1982, agrega que las configuraciones del estomago varían con respecto a la especie. Al parecer estas diferencias están relacionadas directamente con el tipo y tamaño de alimento que consumen.

Paniagua 1983 y Caceci 1997; reportan en peces tres porciones: Zona de cardias o región inicial, cuerpo o región media y zona del piloro o región terminal. Arias 1996, Chávez Y Vazzoler 1984, plantean que el estomago en Semaprochilodus insignis es una estructura de tres porciones así: la primera localizada después del esófago con características enzimáticas y una porción media a manera de bolsa muy distensible que cumple funciones de almacenamiento y fermentación de alimento, estas regiones presentan paredes muy finas; la ultima porción distal es musculosa y potente. Histológicamente el estomago presenta una mucosa con pliegues, formada por el epitelio cilíndrico simple y lamina propia, una submucosa de tejido conectivo laxo areolar y finalmente una túnica muscular que se manifiesta por la presencia de mioblastos. Algunas especies tales como Semaprochilodus insignis, presentan células mucosas que penetran a través de la mucosa epitelial, estas células no fueron observadas en Pterophyllum scalare porque no se realizaron tinciones especiales y pruebas histoquímicas. Florez 2000 y Paniagua 1983, en Trichopterus trichopterus y Semaprochilodus insignis, reportan que la capa muscular de la mucosa tiene una sola capa, lo cual coincide con las observaciones realizadas en Pterophyllum scalare.

Ano: La estructura histológica intestinal suele ser el último tramo digestivo bastante sencillo. En la terminación del intestino se forma el esfínter anal. El ano se abre al exterior por delante del orificio genito- urinario. Los peces teleósteos carecen de cloaca.

Boca: Se pudo observar en cortes transversales que la abertura bucal surge bastante tarde en la vida embrionaria de Pterophyllum scalare), puesto que se forma después de haberse formado los rudimentos de los órganos primarios es decir a las 47 horas 47 minutos en el estadio de esbozo de maxila y premaxila. Además, las estructuras que se forman en la región bucal no siempre son de origen endodérmico, ya que después de la perdida de la membrana faríngea, el ectodermo forma gran parte de su revestimiento. No obstante en este trabajo la cavidad bucal se toma en cuenta porque se encuentra asociada ya sea anatómicamente y fisiológicamente o embriológicamente al sistema digestivo. La invaginación estomodea, además de formar parte del epitelio oral, 
origina células en su región dorsal, las cuales se convierten en una estructura sacular, la bolsa de rathke, que constituye el esbozo del lóbulo anterior de la hipófisis. Esta bolsa se dirige hacia el infundíbulo en el diencéfalo pero por un tiempo permanece abierta y mantiene su conexión con el epitelio oral, se une firmemente al infundíbulo y forma finalmente el lóbulo anterior de la hipófisis o pituitaria.

Al igual que lo reportado para la especie Trichogaster trichopterus, la maxila de Pterophyllum scalare, presenta abundante tejido conectivo, permitiendo cerrar herméticamente la boca. No se observaron glándulas salivares en Pterophyllum scalare, esta observación concuerda con lo reportado por Florez (2000) y Ziswler (1980) quienes indican que en los peces óseos no se conocen glándulas salivares pluricelulares. Esta característica se a que los peces reciben el alimento lo suficientemente húmedo por lo tanto no necesitan glándulas en la primera porción del intestino anterior, para su transporte y posterior digestión a lo largo del tubo digestivo.

Durante la elaboración de este trabajo se encontraron algunos problemas metodológicos; el primero lo constituye la transparencia del embrión, lo cual hace que la observación estereoscópica resulte un poco difícil y al momento de hacer la inclusión en parafina, se dificulta la orientación especialmente de los embriones de menor tamaño. Además debido a la gran cantidad de vítelo que presentan los ovocitos los tejidos tienden a romperse, por lo tanto durante las primeras fases del desarrollo, no se pudieron observar.

\section{AGRADECIMIENTOS}

Universidad Distrital Francisco José de Caldas, por su formación académica y profesional. Pontificia universidad Javeriana por el espacio cedido en el laboratorio. Instituto de acuicultura (IAALL), Universidad de los llanos por permitir acceder al material biológico. Oranda Fish por asesoría y colaboración con el fin de facilitar la obtención de material biológico. Sistemas Integrados de Gestión por su colaboración mediante el uso de equipos informáticos y diseño multimedia.

\section{RESUMEN}

Debido a la poca información sobre el desarrollo de sistemas orgánicos en el pez Pterophylum scalare (Liechtenstein 1823) estudiamos yiempo neto de desarrollo de cada estadio de la organogénesis, tiempo acumulado y diferenciación de estructuras características de cada estadio. Se obtuvo un total de 8 estadios en la organogénesis del sistema digestivo, comprendidos entre la gástrula y la reabsorción total del saco vitelino. La duración de la organogénesis del sistema digestivo fue de 119 horas 44 minutos.

Palabras clave: Organogénesis, estadios, sistema digestivo, desarrollo embrionario, diferenciación celular, peces.

\section{REFERENCIAS}

Ahearn, G., R. Behnke, V. Zonno \& C. Storelli. 1992. Kinetic heterogeneity of Na-D-glucose cotransport in teleost gastrointestinal tract. Am. J. Physiol. 263: R1018- R1023.

Anzola, Escobar \& P. Contreras. 1993. Fundamentos de acuicultura continental. INPA, Bogotá, Colombia. $197 \mathrm{p}$.

Arias, J.A. 1996. Anatomía de la Sapuara Sp. Dahlia 1: $152-156$

Balinsky, B. 1978. Introducción a la Embriología. Omega, S.A., Barcelona, España. 644 p.

Buddington, R.K. \& J. Diamond. 1987. Pyloric ceca of fish a "new" absorptive organ. Am. J. Physiol. 252: G65- G76.

Bruce, M. 1990. Embriología básica de Patten. McGrawHill. México, D.F., México. p. 2-6, 193-195

Caceci, T. 1984. Scanning electron microscopy of goldfish Carassius auratus intestinal mucosa. J. Fish Biol.. 25: 1-12.

Chavez, P. \& G. Vazzoler. 1984 Aspectos biológicos de peixes amazónicos III. Anatomía microscópica do esófago, estomago e cecos pilóricos de Semaprochilodus insignis (Characiformes: prochilodontidae). Acta Amazónica. 14: 343-353.

Cubillos, R. 1994. Estudio embrionario y larval, macroscópico e histológico de Sp: Pterophyllum scalare. Tesis, Universidad Javeriana, Bogotá, Colombia. 
Eslava, P. 1997 Estudio sobre la anatomía macro y microscópica del sistema digestivo de la cachama blanca, Piaractus brachypomus. UNILLANOS/ COLCIENCIAS, Villavicencio, Colombia. 137 p.

Estrada, E., L. Peralta \& P. Rivas. 1982. Manual de Técnicas Histológicas. AGT, México. 140 p.

Florez, N. 2000. Descripción anatómica e histologiaza del sistema digestivo del pez gourami tres manchas Trichogaster trichopterus trichopterus. Universidad Distrital Francisco José de Caldas, Bogotá, Colombia. p. 12-2.

Di Fiore, M. 1953. Diagnóstico Histológico. Vol. 1. Cicaam, Madrid, España. 215 p.

Gisbert, E., A. Rodriguez, F. Castellano \& P. Willliot. 1998. A histological study of the development of the digestive tracto siberian sturgeon (Acipenser bean) during early ontogeny. Aquaculture. 167: 195-209.

Gueimundi, J. 1989. Embriología. Pueblo y educación, La Habana, Cuba.

Gouldin, M. \& M. Carvalo. 1982. Life history and management of the tambaqui (Colossoma macropomun, caracidae), an important amazonian food fish. Rev. Bra. Zool. 1(2): 107-133.

Halvert, J. 1989. Fish Nutrition. Academy, New York, New York, EEUU. 794 p.

Halder, W. 1987. Anatomie der fische. In Principios fundamentals de la alimentación de los peces.. Acribia. Madrid, España. 275 p.

Herbert, A. 1978. Peces tropicales. Hispanoeuropea, Barcelona, España. 272 p.

Hirji, K. 1983. Non-specific carboxilic esterase activity in the digestive tract of the perch. J. Fish Biol. 22: 1-7.

Hussain, A.H. 1953. On the functional morphology of the alimentary tract of some omnivorous fish. Proc. Egyptian Acad. Sci. 4: 17-39.

Kjorsvik, E. \& L. Reiersen. 1991. Histomorphology of the Atlantic halibut (Hippoglossus hippoglossus). An indication of the timing of functionality. Rev. Fish. Soc. Brit. Isles. p. 1-19.

Kjorsvik, E. \& L. Reiersen. 1990. Early development of the digestive tract of cod larvae, Gadus morhua, during start-feeding and starvation. Rev. Fish. Soc. Brit. Isles. p. 1-15.

Luna, G.L. 1968. Manual of histologic staining methods of the armed forces institute of pathology. McGraw-Hill, Toronto, Canada. 320 p.
Mattison, A. \& B. Holstein. 1980. The ultrastucture of the gastric glands and its relation to induced secretory activity of cod, Gadus morhua (Day). Acta Physiol. Escand. 109: 51-60.

Meyer, J. 1993. Nutritional fish diseases. J. Fish. Biol. p. $311-319$.

Morrison, C.M. \& J.R. Wright. 1999. A study of the histology of the digestive tract of the Nile tilapia. J. Fish Biol. 54: 597-606.

Paniagua, R \& M. Nistal. 1983. Introducción a la histología animal y comparada. Labor Universitaria, Madrid, España. p. 339- 503.

Petrovicky, I. 1990. La enciclopedia de los peces de acuario. Susaeta, Madrid, España. 500 p.

Rogers, W. 1979. Anatomy and Histology of the channel catfish. Auburn University, Alabama, EEUU. 94 p.

Romer, J \& A. Pearson. 1966. Anatomía comparada de los vertebrados. Interamericana, México, D.F., México. $512 \mathrm{p}$.

Smith, L.S. 1989. Digestive funtions in teleost fishes. Fish Nutrition.. Academic, San Diego. p. 331-421.

Theodore, W. 1978. Morfogénesis de los vertebrados. Limusa. México. p. 307- 350.

Torres, Rafael. 1993. Manual de cría de peces ornamentales. Naturaleza y vida, Buenos Aires. 350 p.

Ulrich, W. \& V. Storch. 1973. Estudio comparado de la citología e histología animal. Urmo. Barcelona. 361 p.

Velandia, I. 2000. Absorción de nutrientes en intestino anterior y ciegos pilóricos de peces. Universidad Nacional, Bogotá. 145 p.

Verhoef, J. 2002. Enciclopedia de los peces tropicales. Libsa. Madrid. 256 p.

Werner, S. 1987. Principios fundamentales de la alimentación de los peces. Acribia. Madrid. 275 p.

Witcomb, R. \& Clark, S. 1980. The alimentary canal and digestion. Aquaculture. 19: 93-95.

Wolfgang, S. 1990. Cría y reproducción de los peces de acuario. Hispano Europea, Madrid. 465 p.

Zihler, G. 1982. Histology of the stomach of Tilapia nilotica from the river Nile. J. Fish Biol. :25-32.

Ziswiler, V. 1980. Zoología Especial de Vertebrados, Tomo II. Omega, México. 637 p. 